\title{
Clinical Research in Paediatrics: What Can We Do?
}

\author{
Alessandra Pugi ${ }^{1} \cdot$ Valentina Borsi $^{1} \cdot$ Alessio Fabbiano $^{1} \cdot$ Maria Carmela Leo $^{1}$
}

Published online: 9 May 2015

(c) Springer International Publishing Switzerland 2015

\section{Dear Editor,}

We read with great interest the paper 'Ethics of Pharmacological Research Involving Adolescents' by Welisch and Altamirano-Diaz [1].

They well summarized the practical and ethical difficulties in conducting research involving adolescents. Poor evidence for safe and effective drug use in paediatric patients represents a clinical burden. In fact, $70 \%$ of prescribed medications are estimated to be off-label, laying patients open to a high risk of adverse effects [2-4]. Isn't this an ethical issue too?

We agree that pharmacological research should be encouraged. The European Paediatric Regulation [5] aims to improve and support clinical research in paediatrics by strengthening pharmaceutical companies' contribution. Yet it also enforces very strong rules which do not help pharmaceutical companies increase funding in developing drugs for children.

We strongly believe that an important role could be played by independent research. Independent researchers have a real interest in addressing the medical need to get data on safety and efficacy of current medications in the paediatric subset. As Welisch and Altamirano-Diaz [1] argued, the hurdles in performing clinical trials in children and adolescents involve methodological and ethical

This article is part of the topical collection on Ethics of Pediatric Drug Research.

Alessandra Pugi

alessandra.pugi@meyer.it

1 Clinical Trial Office, Anna Meyer Children's Hospital, Viale Pieraccini 24, 50139 Florence, Italy aspects. Therefore, it is of crucial importance to support independent investigators with adequate facilities and tools.

Italy's Tuscany region promoted the creation of Clinical Trial Offices (CTOs) in the region's general and university hospitals. The Anna Meyer Children's Hospital, a third level university hospital, set up a CTO in December 2014 with the aim of promoting clinical research in paediatrics. The team is formed by personnel with different, but complementary, competences: paediatricians, pharmacologists, epidemiologists, biostatisticians, data managers, and nurses. The office supports investigators in designing and planning clinical studies in paediatrics, drafting protocols, improving the delivery of informed consent, and helping researchers to submit their clinical studies to the national Regulatory Authorities and the regional Ethics Committee. It provides for clinical data to be collected in line with patients' privacy and rights, and correctly published. The CTO of the Anna Meyer Children's Hospital has the mission to promote the feasibility of research projects at the local level, also facilitating patient enrollment and ensuring abidance by informed consent rules as regards for-profit clinical trials.

Improving collaboration and networking among paediatric care and research centres is an important achievement to increase patient recruitment, especially for some rare paediatric conditions. In the event of chronic diseases, there has been an interesting development regarding networking between paediatric and adult healthcare facilities. The transition from child-centered to adult-oriented healthcare systems is a major life event for young people from a clinical point of view. Transition clinics offer a chance for specialized clinical care for adolescents, as well as providing an opportunity for research. Carrying out clinical trials in such environments could offer the opportunity to take care of adolescents and children for a long 
period of time and obtain data about the natural history of diseases and long-term safety profiles of interventions. Finally, this could plug the gap between childhood and adulthood in terms of safety and efficacy information. Transition is an integrated process involving healthcare providers, family and patients; a more conscious informed consent by adolescents and parents could be obtained in this context.

Children have the same rights as adults to receive safe and effective medicines. All efforts should be made in order to promote safe and meaningful pharmacological research in paediatrics. In this context, we strongly believe that independent research can have an important role and should be encouraged and supported by specific bodies, like CTOs.

Conflicts of interest A. Pugi, V. Borsi, A. Fabbiano and M. C. Leo declare no conflicts of interest.

\section{References}

1. Welisch E, Altamirano-Diaz LA. Ethics of pharmacological research involving adolescents. Paediatr Drugs. 2015;17(1):55-9. doi:10.1007/s40272-014-0114-0.

2. Horen B, Montastruc JL, Lapeyre-Mestre M. Adverse drug reactions and off-label drug use in paediatric outpatients. $\mathrm{Br} \mathrm{J}$ Clin Pharmacol. 2002;54(6):665-70.

3. Chai G, Governale L, McMahon AW, Trinidad JP, Staffa J, Murphy D. Trends of outpatient prescription drug utilization in US children, 2002-2010. Pediatrics. 2012;130(1):23-31. doi:10.1542/ peds.2011-2879.

4. Palmaro A, Bissuel R, Renaud N, Durrieu G, Escourrou B, Oustric S, Montastruc JL, Lapeyre-Mestre M. Off-label prescribing in pediatric outpatients. Pediatrics. 2015;135(1):49-58. doi:10.1542/ peds.2014-0764.

5. Regulation (EC) No 1901/2006 of the European Parliament and of the Council of 12 December 2006 on medicinal products for paediatric use. 2006. http://ec.europa.eu/health/files/eudralex/vol1/reg_2006_1901/reg_2006_1901_en.pdf. Accessed 4 May 2015. 